\title{
Hypercyclictty and Countable Hypercyclicity for Adjoint of $\theta-$ Operators
}

Buthainah A. Ahmed*

Date of acceptance $28 / 2 / 2010$
Hiba F. Al-Janaby*

Abstract

Let $H$ be an infinite dimensional separable complex Hilbert space and let $T \in B(H)$, where $B(H)$ is the Banach algebra of all bounded linear operators on $H$. In this paper we prove the following results.

If $T \in B(H)$ is a $\theta$-operator, then

1. $T^{*}$ is a hypercyclic operator if and only if $\sigma\left(\left.T\right|_{M}\right) \cap D \neq \phi$ and $\sigma\left(\left.T\right|_{M}\right) \cap(C \backslash \bar{D}) \neq \phi$ for every hyperinvariant subspace $M$ of $T$.

2. If $T$ is a pure, then $T^{*}$ is a countably hypercyclic operator if and only if $\sigma\left(\left.T\right|_{M}\right) \cap(C \backslash \bar{D}) \neq \phi$ and $\sigma(T) \cap D \neq \phi$ for every hyperinvariant subspace $M$ of $T$.

3. $T^{*}$ has a bounded set with dense orbit if and only if for every hyperinvariant subspace $M$ of $T, \sigma\left(\left.T\right|_{M}\right) \cap(C \backslash \bar{D}) \neq \phi$.

Keywords: $\theta$-operator, hypercyclic, countably hypercyclic, single valued extension property (SVEP), Bishop's property $(\beta)$, decomposition property $(\delta)$.

\section{Introduction}

Let $H$ be an infinite dimensional separable complex Hilbert space, and $\mathrm{B}(H)$ be the set of all bounded linear operators on $H$, we denote as usual the spectrum, the point spectrum and the approximate point spectrum of $T$ by $\sigma(T), \quad \sigma_{p}(T)$ and $\sigma_{a p}(T)$. Following [1], the $\operatorname{Lat}(T)$, where $T \in \mathrm{B}(H)$, denoted the collection of all $T$ - invariant closed linear subspaces of $H$. If $T \in \mathrm{B}(H)$ and $M \in \operatorname{Lat}(T)$, then $\left.T\right|_{M} \in \mathrm{B}(M)$ is the restriction of $T$ to $M$.

An operator $T \in \mathrm{B}(H)$ is called $\theta$-operator if $T^{*} T$ commutes with $T+T^{*}$, [2]. Recall that $T \in \mathrm{B}(H)$ is called normaloid if $r(T)=\|T\|$, where $r(T)=\sup \{|\lambda|: \lambda \in \sigma(T)\},[3]$.

It is well known [4] that

$\theta$-operator $\Rightarrow$ normaloid

An operator $T \in \mathrm{B}(H)$ is called hyponormal if $\left\|T^{*} x\right\| \leq\|T x\|$ for all $x \in H$. Campbell and Gellar [5] gave an example of a $\theta$-operator which is not hyponormal, also Al-Sultan [6] gave an example of an operator which is hyponormal but it is not $\theta$-operator.

If $T \in \mathrm{B}(H)$ and $x \in H$, then the orbit of $x$ under $T$ is $\operatorname{Orb}(T, x)=\left\{x, T x, T^{2} x, \ldots\right\}, \quad$ [7]. If

*Department of Mathematics - College of Science - University of Baghdad 
$E \subseteq H$, then the orbit of $E$ under $T$ is

$\operatorname{Orb}(T, E)=\bigcup\left\{E, T(E), T^{2}(E), \ldots\right\}=$ $\bigcup_{x \in E} \operatorname{Orb}(T, x),([8],[9])$.

An operator $T \in \mathrm{B}(H)$ is called hypercyclic if there is a vector $x \in H$ with dense orbit $\left\{x, T x, T^{2} x, \ldots\right\}$, [7].

Following ([8], [9]), we say that an operator $T \in \mathrm{B}(H)$ is countably hypercyclic if there exists a bounded, countable, separated set $\mathrm{E}$ with dense orbit. Recall that a set $\mathrm{E} \subseteq H$ is separated if there exists an $\varepsilon>0$ such that $\|x-y\| \geq \varepsilon$ for all $x, y \in \mathrm{E}$ with $x \neq y$.

In [7], Feldman, Miller, and Miller proved that the cohyponormal operators (the adjoint of hyponormal operators) are hypercyclic if and only if $\quad \sigma\left(\left.T\right|_{M}\right) \cap D \neq \phi \quad$ and $\sigma\left(\left.T\right|_{M}\right) \cap(C \backslash \bar{D}) \neq \phi \quad$ for every hyperinvariant subspace $M$ of $T$. Recently Feldman [8] showed that there are countably hypercyclic operators which are not hypercyclic. Furthermore, Feldman showed that the pure cohyponormal operators are countably hypercyclic if and only if $\sigma\left(\left.T\right|_{M}\right) \cap(C \backslash \bar{D}) \neq \phi \quad$ and $\sigma(T) \cap D \neq \phi$ for every hyperinvariant subspace $M$ of $T$. In this paper we give an example of a $\theta$-operator which is not hypercyclic and prove that the adjoint of $\theta$-operator is hypercyclic if and only if $\sigma\left(\left.T\right|_{M}\right) \cap D \neq \phi \quad$ and $\sigma\left(\left.T\right|_{M}\right) \cap(C \backslash \bar{D}) \neq \phi \quad$ for every hyperinvariant subspace $M$ of $T$. We also give an example of a $\theta$-operator which is not countably hypercyclic and prove that the adjoint of pure $\theta$-operator is countably hypercyclic if and only if $\sigma\left(\left.T\right|_{M}\right) \cap(C \backslash \bar{D}) \neq \phi$ and $\sigma(T) \cap D \neq \phi$ for every hyperinvariant subspace $M$ of $T$. Finally we prove the adjoint of $\theta$-operator has bounded set with dense orbit if and only if for every hyperinvariant subspace $M$ of $T, \sigma\left(\left.T\right|_{M}\right) \cap(C \backslash \bar{D}) \neq \phi$.

\section{Preliminaries}

An operator $T \in \mathrm{B}(H)$ is said to have single valued extension property (SVEP) at $\lambda_{0}$ if

for every open set $U \subseteq C$ containing $\lambda_{0}$ the only analytic solution $f: U \rightarrow H$ of the equation

$$
\left(T-\lambda_{0}\right) f(\lambda)=0 \quad\left(\lambda_{0} \in U\right)
$$

is the zero function [1]. An operator $T$ is said to have SVEP if $T$ has SVEP at every $\lambda \in C$.

Given $T \in \mathrm{B}(H)$, the local resolvent set $\rho_{T}(x)$ of $T$ at the point $x \in H$ is defined as the union of all open subsets $U \subseteq C$ for which there is an analytic function $f: U \rightarrow H$ such that

$$
(T-\lambda) f(\lambda)=x \quad(\lambda \in U)
$$

The local spectrum $\sigma_{T}(x)$ of $T$ at $x$ is then defined as $\sigma_{T}(x)=C \backslash \rho_{T}(T)$

For $T \in \mathrm{B}(H)$, we define the local ( resp. glocal ) spectral subspaces of $T$ as follows. Given a set $F \subseteq C$ ( resp. a closed set $G \subseteq C$ ).

$H_{T}(F)=\left\{x \in H: \sigma_{T}(x) \subseteq F\right\}$

( resp.

$\mathrm{H}_{T}(F)=\{x \in H:$ there exists an analytic function $f: C \backslash G \rightarrow H$ such that $(T-\lambda) f(\lambda)=x$ for all $\lambda \in C \backslash G\})$.

Note that $T$ has SVEP if and only if $H_{T}(F)=\mathrm{H}_{T}(F)$ for all closed sets $F \subseteq C$, [1, Proposition (3.3.2)]. 
If $U \subseteq C$ is an open set, then define $H_{T}(U)=\bigcup\left\{H_{T}(F): F \subseteq U\right.$ is compact\}. $H_{T}(U)$ contains all eigenvectors for $T$ whose eigenvalues belong to $U$ and that $H_{T}(U)$ is a hyperinvariant subspace for $T$, althought it is not necessarily closed, [8].

An operator $T \in \mathrm{B}(H)$ has Dunford's property (C) if the local spectral subspace $H_{T}(F)$ is closed for every closed set $F \subseteq C$. An operator $T \in \mathrm{B}(H)$ is said to has Bishop's property $(\beta)$ if for every sequence $f_{n}: U \rightarrow H \quad$ such that $(T-\lambda) f_{n}(\lambda) \rightarrow 0 \quad$ uniformly on compact subsets in $U$, it follows that $f_{n} \rightarrow 0$ uniformly on compact subsets in $U$. It is well known [1] that

Bishop's property $(\beta) \Rightarrow$ Dunford's property $(C) \Rightarrow$ SVEP

Moreover, an operator $T \in \mathrm{B}(H)$ has decomposition property $(\delta)$ if $H=\mathrm{H}_{T}(\bar{U})+\mathrm{H}_{T}(\bar{V})$ for every open cover $\{U, V\}$ of $C$.

As shown in [1], an operator $T \in \mathrm{B}(H)$ has property $(\delta)$ iff it is the quotient of a decomposable operator. Moreover properties $(\beta)$ and $(\delta)$ are dual to each other, in the sense that an operator $T \in \mathrm{B}(H)$ has property $(\beta)$ iff its adjoint has property $(\delta)$, and conversely, $T$ has property $(\delta)$ iff its adjoint has property $(\beta)$.

Proposition 2.1. [1] Suppose that the operator $T \in \mathrm{B}(H)$ on the Hilbert space $H$ has SVEP, and that $F \subseteq C$ is a closed set for which the space $H_{T}(F)$ is closed. Then $\sigma\left(\left.T\right|_{H_{T}\{F)}\right) \subseteq F \cap \sigma_{T}(x)$

The following result from Feldman, Miller and Miller [7], gives the relation between parts of the spectrum and the local spectra of an operator with Dunford's property (C).

Proposition 2.2. [7] If $T \in \mathrm{B}(H)$ has Dunford's property (C), then $\sigma_{T}(x)=\sigma\left(\left.T\right|_{H_{T}(F)}\right) \quad$ whenever $F=\sigma_{T}(x)$ for some nonzero $x \in H$.

The following result from Feldman, Miller and Miller [7], gives sufficient condition for an operator to be hypercyclic, we denote the interior and exterior of the unit circle by $D, C \backslash \bar{D}$ respectively.

Corollary 2.3. [7] Let $H$ be a complex Hilbert space and suppose that $T \in \mathrm{B}(H)$ has the decomposition property $(\delta)$. If $\sigma_{T^{*}}(x) \cap D \neq \phi$ and $\sigma_{T *}(x) \cap(C \backslash \bar{D}) \neq \phi \quad$ for every nonzero $x \in H$. Then $T$ is hypercyclic.

The following result from Feldman [8], gives sufficient condition for an operator to be countable hypercyclic.

Theorem 2.4. [8] (The Countably Hypercyclic Criterion) Suppose that $T \in \mathrm{B}(H)$. If there exists two subspaces $Y$ and $Z$ in $H$, where $Y$ is infinite dimensional and $Z$ is dense in $H$ such that

$$
\begin{aligned}
& \text { 1. } T^{n} x \rightarrow 0 \text { for every } x \in Y \text {, and } \\
& \text { 2. There exists functions } \\
& B_{n}: Z \rightarrow H \text { such that } \\
& T^{n} B_{n}=\left.I\right|_{Z} \text { and } B_{n} x \rightarrow 0 \text { for } \\
& \text { every } x \in Z
\end{aligned}
$$

Then $T$ is countably hypercyclic.

Theorem 2.5. [8] Suppose that $T \in \mathrm{B}(H)$ If $\mathrm{H}_{T}(D)$ is infinite dimensional and $\mathrm{H}_{T}(C \backslash \bar{D})$ is dense, then $T$ is countably hypercuclic. 
Proposition 2.6. [8]

a. If $T \in \mathrm{B}(H)$ and there is a bounded set $E$ with $\operatorname{Orb}(T, E)$ dense, then sup $\left\|T^{n}\right\|=\infty$.

b. If there is a set $E$ that is bounded away from zero and $\operatorname{Orb}(T, E)$ is dense, then $T$ cannot be expensive, that is there exists an $x \in H$ such that $\|T x\|<\|x\|$.

In what follows, $B(a, r)$ will denote the open ball at $a$ with radius $r$, where for $a \in H$ and $r>0$.

\section{Remark 2.7.}

a. Notice that if $T$ is countably hypercuclic and $E=\left\{x_{n}\right\}$ a bounded separated sequence with dense orbit, then one may assume that $x_{n} \neq 0$ for all $n$, thus it follows that $E$ is both bounded and bounded away from zero, [8].

b. If an operator $T$ has a set with dense orbit, then any non-zero multiple of that set also has dense orbit. Thus $T$ has a bounded set with dense orbit if and only if the unit ball has dense orbit if and only if $B(a, r)$ has dense orbit for any $r>0,[8]$.

\section{Hypercyclicity}

It is well known that the restriction of $\theta$-operator $\left.T\right|_{M}$ is a $\theta$-operator for every $M \in \operatorname{Lat}(T)$, and if $T$ is a $\theta$-operator and invertiable, then $T^{-1}$ is a $\theta$-operator, [7]. Recall that an operator $T \in \mathrm{B}(H)$ is dominant if $(T-\lambda) H \subset(T-\lambda)^{*} H$ for all scalars $\lambda, \mathrm{Y}$. kato show that every $\theta$-operator is dominant, [10]

Before proving one of important results in this paper, we need the following.
Definition 3.1. [11] An operator $T \in \mathrm{B}(H)$ is said to have the property (II) if for every $\lambda, \mu \in \sigma_{a p}(T)$ and every bounded sequences of vectors $x_{n}$ and $y_{n}$ such that $\lambda \neq \mu$ and $\left\|(T-\lambda) x_{n}\right\| \rightarrow 0, \quad\left\|(T-\lambda) y_{n}\right\| \rightarrow 0$, the sequence $\left\langle x_{n}, y_{n}\right\rangle$ converges to 0 as $n \rightarrow \infty$.

Theorem 3.2. [11] If $T$ has property (II), then $T$ also has property $(\beta)$.

It is well known that dominant operator has Bishop's property $(\beta)$ but couldn't find the proof, so we prove it.

Theorem 3.3. Every dominant operator has Bishop's property $(\beta)$.

Proof. Let $\lambda, \mu \in \sigma_{a p}(T) \quad(\lambda \neq \mu)$ and sequences $\left\{x_{n}\right\},\left\{y_{n}\right\}$ of bounded vectors in $H$ satisfy $\left\|(T-\lambda) x_{n}\right\| \rightarrow 0$, $\left\|(T-\lambda) y_{n}\right\| \rightarrow 0 \quad($ as $n \rightarrow 0)$. Since $T$ is dominant, then $\left\|(T-\lambda)^{*} y_{n}\right\| \rightarrow 0$ as $n \rightarrow \infty$. Hence

$(\lambda-\mu)\left\langle x_{n}, y_{n}\right\rangle=\left\langle(\lambda-T) x_{n}, y_{n}\right\rangle+\left\langle x_{n},(T-\mu)^{\circ} y_{n}\right\rangle \rightarrow 0$ as $(n \rightarrow 0)$

This implies that $\left\langle x_{n}, y_{n}\right\rangle \rightarrow 0$. Then $T$ has the property (II) Therefore $T$ has property $(\beta)$ by Theorem (3.2). 口

Remark 3.4. Every $\theta$-operator has Bishop's property $(\beta)$.

Now we give an example of $\theta$-operator which is not hypercyclic, We begin with the following result.

Corollary 3.5. [12] If $T \in \mathrm{B}(H)$ and $\|T\| \leq 1$, then $T$ is not hypercyclic. 
Example 3.6. Let $U$ be the unilateral shift operator defined on $\ell^{2}(\mathrm{~N})$.

$U\left(x_{1}, x_{2}, x_{3, \ldots}\right)=\left(0, x_{1}, x_{2}, x_{3}, \ldots\right)$

One can easily cheek that $\left(\left(U^{*} U\right)\left(U+U^{*}\right)\right)\left(x_{1}, x_{2}, x_{3}, \ldots\right)=$ $\left(x_{2}, x_{1}+x_{3}, x_{1}+x_{4}, \ldots\right)$

$\left(\left(U+U^{*}\right)\left(U^{*} U\right)\right)\left(x_{1}, x_{2}, x_{3}, \ldots\right)=$ $\left(x_{2}, x_{1}+x_{3}, x_{1}+x_{4}, \ldots\right)$

Which implies $U$ is a $\theta$-operator.

Since $U$ is not hypercyclic by

Corollary (3.5).

Now we give our Theorem.

Theorem 3.7. If $T$ is a $\theta$-operator on a separable Hilbert space $H$, then $T^{*}$ is hypercyclic if and only if $\sigma_{T}(x) \cap D \neq \phi \quad$ and $\sigma_{T}(x) \cap(C \backslash \bar{D}) \neq \phi$ for every nonzero $x \in H$.

Proof. If $T$ is a $\theta$-operator on $H$, then $T$ has property $(\beta)$ by Remark (3.4). Thus $T$ has property (C), and so $T^{*}$ has property $(\delta)$. If the local spectra $\quad \sigma_{T}(x) \cap D \neq \phi \quad$ and $\sigma_{T}(x) \cap(C \backslash \bar{D}) \neq \phi$ for every nonzero $x \in H$, then $T^{*}$ is hypercyclic by Corollary (2.3).

Conversely, suppose that $T^{*}$ is hypercyclic. First we prove that every part of the spectrum of $T$ meets both $D$ and $C \backslash \bar{D}$, i.e., $\sigma\left(\left.T\right|_{M}\right) \cap D \neq \phi$ and $\sigma\left(\left.T\right|_{M}\right) \cap(C \backslash \bar{D}) \neq \phi$.

Let $\quad S=\left.T\right|_{M}$ for some $M \in \operatorname{Lat}(T) \backslash\{0\}$. If $x$ is a hypercyclic vector for $T^{*}$, then by the definition of hypercyclic vector

$\operatorname{Orb}\left(T^{*}, x\right)=\left\{x, T^{*} x,\left(T^{*}\right)^{2} x, \ldots\right\} \quad$ is dence in $H$

We claim the projection $P_{M} x$ is hypercyclic for $S^{*}=\left.P_{M} T^{*}\right|_{M}$. Since $M \in \operatorname{Lat}(T) \backslash\{0\}$, then by Corollary of Theorem 2, [3, P.39], $P_{M} T P_{M}=T P_{M}$. Consequently
$P_{M} T^{*} P_{M}=P_{M} T^{*}, \quad$ and

$S^{*}\left(P_{M} x\right)=\left(\left.P_{M} T^{*}\right|_{M}\right)\left(P_{M} x\right)=P_{M} T^{*}\left(P_{M} x\right)$

$=\left(P_{M} T^{*} P_{M}\right)(x)=P_{M} T^{*}(x)=P_{M}\left(T^{*} x\right)$

New a little bit calculation show that

$$
\begin{aligned}
& \overline{\operatorname{Ork}\left(S^{*}, P_{M}(x)\right)}=\overline{\left\{P_{M}(x), S^{*}\left(P_{M} x\right),\left(S^{*}\right)^{2}\left(P_{M} x\right), \ldots\right\}} \\
& =\left\{\overline{\left.P_{M}(x), P_{M}\left(T^{*} x\right), S^{*}\left(P_{M} T^{*} x\right), \ldots\right\}}\right. \\
& =\left\{\overline{\left.P_{M}(x), P_{M} T^{*}(x), P_{M}\left(T^{*}\right)^{2}(x), \ldots\right\}}\right. \\
& =P_{M}\left\{\overline{\left.x, T^{*} x,\left(T^{*}\right)^{2} x, \ldots\right\}}=\overline{P_{M}(H)}=M\right. \\
& \text { i.e., the projection } P_{M} x \text { is }
\end{aligned}
$$
hypercyclic for $S^{*}=\left.P_{M} T^{*}\right|_{M}$. Since $S$ is a $\theta$-operator, then $r(S)=\|S\|=\left\|S^{*}\right\|>1 \quad$ [ If $\left\|S^{*}\right\| \leq 1$, then $S^{*}$ is not hypercyclic this is impossible].

$$
\text { We prove } \sigma(S) \cap(C \backslash \bar{D}) \neq \phi \text {. }
$$

Since $\quad r(S)=\sup \{|\lambda|: \lambda \in \sigma(S)\}>1$, this means that $\sigma(S)$ contains a complex number $\lambda$ such that $|\lambda|>1$ and since $C \backslash \bar{D}=\{\lambda:|\lambda|>1\}$. Consequently $\sigma(S) \cap(C \backslash \bar{D}) \neq \phi$.

$$
\text { Now to show that }
$$
$\sigma(S) \cap D \neq \phi$. If $\sigma(S) \subset(C \backslash \bar{D})$. i.e, $\sigma(S) \cap D=\phi$, then for all $\lambda$ in $\sigma(S)$ is nonzero and hence $0 \in \rho(S)$, thus $S$ is an invertiable and therefore $S^{-1}$ is a $\theta$-operator.

Since $\sigma(S)$ contains a complex number $\lambda$ such that $|\lambda|>1$, then by [3, P.171], $\sigma\left(S^{-1}\right)$ contains a complex number $\lambda^{-1}$ such that $|\lambda| \leq 1$. Thus $r\left(S^{-1}\right)=\inf \left\{|\lambda|: \lambda^{-1} \in \sigma(S)\right\} \leq 1$.

Consequently $\left\|S^{-1}\right\| \leq 1$. But $S^{*}$ hypercyclic and invertiable, which implies that $\left(S^{*}\right)^{-1}$ is hypercyclic and thus $\left\|\left(S^{*}\right)^{-1}\right\|>1$ by Corollary (3.5). 
Notice that $\left\|S^{-1}\right\|=\left\|\left(S^{-1}\right)^{*}\right\|=\left\|\left(S^{*}\right)^{-1}\right\|>1$, this is a contradiction since $\left\|S^{-1}\right\| \leq 1$, it follows that $\sigma(S) \cap D \neq \phi$.

Since $T$ is a $\theta$-operator, then $T$ has property $(\beta)$ by Remark (3.4) and hence $T$ has property (C). Thus by Proposition (2.2), $\sigma_{T}(x)=\sigma\left(\left.T\right|_{H_{T}(F)}\right)$ whenever $F=\sigma_{T}(x)$ for every nonzero $x$ and as in the previous paragraph, it follows that

$\sigma_{T}(x) \cap D \neq \phi \quad$ and

$\sigma_{T}(x) \cap(C \backslash \bar{D}) \neq \phi$

for every nonzero $x \in H$.

view of Proposition (2.2), an equivalent way to state Theorem (3.1) is as follows.

Theorem 3.8. If $T$ is a $\theta$-operator on a separable Hilbert space $H$, then $T^{*}$ is hypercyclic if and only if $\sigma\left(\left.T\right|_{M}\right) \cap D \neq \phi \quad$ and $\sigma\left(\left.T\right|_{M}\right) \cap(C \backslash \bar{D}) \neq \phi \quad$ for every hyperinvariant subspace $M$ of $T$.

\section{Countably Hypercyclicity}

It was shown in [6] that if $T$ is a $\theta$-operator, then for fixed scalar, $\operatorname{ker}(T-\lambda)$ reduces $T$ and $\left.T\right|_{\operatorname{ker}(T-\lambda)}$ is normal. Recall that an operator $T \in \mathrm{B}(H)$ is called pure if there is no reducing subspace $M$ such that $\left.T\right|_{M}$ is normal.

Proposition 4.1. If $T$ is a pure $\theta$-operator, then $T$ has no eigenvahues.

Proof. If $\lambda \in \sigma_{p}(T)$, then $\left.T\right|_{\operatorname{ker}(T-\lambda)}$ is normal, it is a contradiction to definition of pure. Therefore $\sigma_{p}(T)=\phi$.
Now we give an example of $\theta$-operator which is not countably hypercyclic.

Example 4.2. Let $U$ be the unilateral shift operator defined on $\ell^{2}(\mathrm{~N})$

$U\left(x_{1}, x_{2}, x_{3, \ldots}\right)=\left(0, x_{1}, x_{2}, x_{3}, \ldots\right)$

$U$ is a $\theta$-operator by Example (3.6). since $\|U\|=1$, then $\left\|U^{n}\right\| \leq\|U\|^{n}=1$ and hence sup $\left\|U^{n}\right\|<\infty$. Thus can not exists a bounded set $E$ with $\operatorname{Orb}(U, E)$ dence by part (a) of Proposition (2.6). Therefore $U$ is not countably hypercyclic. $\square$

Lemma 4.3.

a. If $T$ is a $\theta$-operator on $a$ Hilbert space $H$, then for any open set $U \subseteq C$, we have $H_{T} \cdot(U)^{\perp}=H_{T}(C \backslash U)$.

b. If $T$ is a pure $\theta$-operator for which $H_{T^{*}}(D)$ is finite dimensional, $H_{T^{*}}(D)=\{0\}$

Proof.

a. Since $T$ is $\theta$-operator, then $T$ has property $(\beta)$ by Remark (3.4), and hence $T^{*}$ has property $(\delta)$. Therefore by $[\mathbf{1}$, Proposition (2.5.14)], for any open set $U \subseteq C$, we have $H_{T^{*}}(U)^{\perp}=H_{T}(C \backslash U)$.

b. Suppose that $H_{T^{*}}(D)$ is a nonzero and finite dimensional. Since $H_{T^{*}}(D)$ is finite dimensional invariant subspace for $T^{*}$, it follows that $T^{*}$ has eigenvectors with eigenvalues in $D$. Let $\lambda$ be such an eigenvalue, then since $\operatorname{ker}\left(T^{*}-\lambda\right) \subseteq H_{T^{*}}(D)$, it follows that $\operatorname{ker}\left(T^{*}-\lambda\right)$ is finite dimensional. Thus by [1, Lemma (3.1.2)], $(T-\bar{\lambda})$ has closed range. Since $T$ is pure, then by Proposition (4.1), $T-\bar{\lambda}$ is one to 
one with closed range, hence $\bar{\lambda} \in\left[\sigma(T) \backslash \sigma_{a p}(T)\right] . \quad$ However, $\left[\sigma(T) \backslash \sigma_{a p}(T)\right]$ is an open set and since $\lambda \in D \cap\left[\sigma(T) \backslash \sigma_{a p}(T)\right]$, it follows that $D \cap\left[\sigma(T) \backslash \sigma_{a p}(T)\right]$ is a non-empty open set. Hence for each $\mu \in D \bigcap\left[\sigma(T) \backslash \sigma_{a} p(T)\right]$ we have $\operatorname{ker}\left(T^{*}-\bar{\mu}\right) \neq\{0\} \quad$ and $\operatorname{ker}\left(T^{*}-\bar{\mu}\right) \subseteq H_{T^{*}}(D)$. It follows that $H_{T^{*}}(D)$ is infinite dimensional, a contradiction. 口

Theorem 4.2. If $T$ is a pure $\theta$-operator on a separable Hilbert space $H$, then $T^{*}$ is countably hypercyclic if and only if for every hyperinvariant subspace $M$ of $T$, $\sigma\left(\left.T\right|_{M}\right) \cap(C \backslash \bar{D}) \neq \phi$ and $\sigma(T) \cap D \neq \phi$

Proof. Suppose the spectral conditions are satisfied. We want to apply Theorem (2.5). So, suppose that $H_{T^{*}}(D)=\{0\}$. Since $T$ is $\theta$-operator, then by part (a) of Lemma (4.1), $H_{T^{*}}(D)^{\perp}=H_{T}(C \backslash D)$, it follows that $H_{T}(C \backslash D)=H$. Thus by Proposition (2.1), $\sigma(T)=\sigma\left(\left.T\right|_{H_{T}(C D)}\right) \subseteq(C \backslash D)$ a contradiction. So, $H_{T^{*}}(D) \neq\{0\}$, now by part (b) of Lemma (4.1) $H_{T^{*}}(D)$ is infinite dimensional. Now, suppose that $H_{T} \cdot(C \backslash \bar{D})$ is not dense in $H$, i.e., $\overline{H_{T^{*}}(C \backslash \bar{D})} \neq H$, then $H_{T^{*}}(C \backslash \bar{D}) \neq H$. Thus $H_{T}(\bar{D})$ is a nonzero I If $H_{T}(\bar{D})=0$, then by part (a) of Lemma $H_{T} \cdot(C \backslash \bar{D})^{\perp}=H_{T}(\bar{D})=0$, and hence $H_{T^{*}}(C \backslash \bar{D})^{\perp}=H$. So $\overline{H_{T^{*}}(C \backslash \bar{D})}=H$. contradicting our assumption]. Therefore $H_{T}(\bar{D})$ is a nonzero hyperinvariant subspace for $T$. Furthermore $\sigma\left(\left.T\right|_{H_{T}(\bar{D})}\right) \subseteq \bar{D}$ contradicting our assumption. Thus it follows that $\mathrm{H}_{T^{*}}(C \backslash \bar{D})$ is dense. So, by Theorem (2.5), $T^{*}$ is countably hypercyclic.

Conversely, suppose $T^{*}$ is countably hypercyclic. Let $E$ be a bounded set, that is bounded away from zero, with dense orbit by part (a) of Remark (2.7). Let $M$ be an invariant subspace for $T$ and let $P_{M}$ be the projection onto $M$. It is easy to prove $\left(\left.T\right|_{M}\right)^{*} P_{M}=P_{M} T^{*}$ and $P_{M}(E)$ is bounded set.

Now

$$
\begin{aligned}
& \overline{\operatorname{Orb}\left(\left(\left.T\right|_{M}\right)^{*}, P_{M}(E)\right)} \\
& =\bigcup_{\substack{P_{\mathcal{X}}(x) \in P_{\mathcal{X}}(E) \\
x \in \mathbb{B}^{*}}} \overline{\left.P_{M}(x),\left(\left.T\right|_{M}\right)^{*}\left(P_{M}(x)\right),\left(\left(\left.T\right|_{M}\right)^{*}\right)^{2}\left(P_{M}(x)\right), \ldots\right\}} \\
& =\bigcup_{\substack{P_{M^{\prime}}(x) \in R_{H^{\prime}}(B) \\
\text { set }}} \overline{\left.P_{M}(x),\left(\left(\left.T\right|_{M}\right)^{*} P_{M}\right)(x),\left(\left(\left.T\right|_{M}\right)^{*}\left(\left.T\right|_{M}\right)^{*} P_{M}\right)(x) \ldots\right\}} \\
& =\bigcup_{\substack{P_{M A}(x) \in P_{M}(E) \\
x \in E^{*}}} \overline{\left\{P_{M}(x),\left(P_{M} T^{*}\right)(x),\left(\left.T\right|_{M}\right)^{*}\left(\left(P_{M} T^{*}\right)(x)\right), \ldots\right\}} \\
& =\bigcup_{\substack{P_{M}(x) \in P_{M}(E) \\
x \in E}} \overline{\left\{P_{M}(x), P_{M}\left(T^{*} x\right),\left(\left(P_{M} T^{*}\right) T^{*}\right)(x), \ldots\right\}} \\
& =\bigcup_{\substack{P_{M}(x) \in P_{M}(E) \\
s \in E}} \overline{\left.\left\{P_{M}(x), P_{M}\left(T^{*} x\right),\left(P_{M} T^{*}\right)^{2}\right)(x), \ldots\right\}} \\
& =P_{M}\left(\bigcup_{x \in E} \overline{\left.\left\{x, T^{*} x,\left(T^{*}\right)^{2} x, \ldots\right\}\right)}=\overline{P_{M}(H)}=M\right.
\end{aligned}
$$


$\sup \left\|\left(\left(\left.T\right|_{M}\right)^{*}\right)^{n}\right\|<\infty$. This is impossible by part (a) of Proposition (2.6)].

Since $T$ is $\theta$-operator, then $\left.T\right|_{M}$ is $\theta$-operator and hence $\left.T\right|_{M}$ is normoliad. Thus $r\left(\left.T\right|_{M}\right)=\left\|\left.T\right|_{M}\right\|>1$. Since

$r\left(\left.T\right|_{M}\right)=\sup \left\{|\lambda|: \lambda \in \sigma\left(\left.T\right|_{M}\right)\right\}>1$, then there is $\lambda \in \sigma\left(\left.T\right|_{M}\right)$ such that $|\lambda|>1$, also since $C \backslash \bar{D}=\{\lambda:|\lambda|>1\}$. So $\sigma\left(\left.T\right|_{M}\right) \cap(C \backslash \bar{D}) \neq \phi$.

Now, if $\sigma(S) \cap D=\phi$, i.e., $\sigma(S) \subset(C \backslash D)$, then for all $\lambda$ in $\sigma(T)$ is nonzero and hence $0 \in \rho(T)$, thus $T$ is an invertiable and therefore $T^{-1}$ is $\theta$-operator. Since $\sigma(T)$ contains a complex number $\lambda$ such that $|\lambda|>1$, then by [3, P.171], $\sigma\left(T^{-1}\right)$ contains a complex number $\lambda^{-1}$ such that $\quad|\lambda| \leq 1$. Thus $r\left(T^{-1}\right)=\inf \left\{|\lambda|: \lambda^{-1} \in \sigma(T)\right\} \leq 1$.

Consequently $\quad\left\|\left(T^{*}\right)^{-1}\right\|=\left\|T^{-1}\right\| \leq 1$, hence $\left\|T^{*} x\right\| \geq\|x\|$ for all $x \in H$, contradicting part (b) of Proposition (2.6)

$\square$

Proposition 4.3. If $T$ is a $\theta$-operator,then $T^{*}$ has a bounded set with dense orbit if and only if for every hyperinvariant subspace $M$ of $T, \sigma\left(\left.T\right|_{M}\right) \cap(C \backslash \bar{D}) \neq \phi$.

Proof. Suppose that every hyperinvariant subspace $M$ of $T$, $\sigma\left(\left.T\right|_{M}\right) \cap(C \backslash \bar{D}) \neq \phi$, we want to show $H_{T^{*}}(C \backslash \bar{D})$ is dense in $H$. So, suppose that $H_{T^{*}}(C \backslash \bar{D})$ is not dense in $H$, i.e., $\overline{H_{T^{*}}(C \backslash \bar{D})} \neq H$, then $H_{T^{*}}(C \backslash \bar{D}) \neq H$ and hence $H_{T}(\bar{D})$ is a nonzero hyperinvariant subspace for
T. Furthermore $\quad \sigma\left(\left.T\right|_{H_{T}(\bar{D})}\right) \subseteq \bar{D}$ contradicting our assumption. Thus $H_{T^{*}}(C \backslash \bar{D})$ is dense in $H$. It follows that if $Z=H_{T^{*}}(C \backslash \bar{D})$, then condition(2) of the Countably Hypercyclic Criterion is satisfied, see [7, Theorem 3.2]. However, condition (2) of the Countably Hypercyclic Criterion easily implies that the unit ball has dense orbit, then by part (b) of Remark (2.7) has a bounded set with dense orbit. The converse is similar to the proof of Theorem (4.2) 口

\section{References}

1. Laursen, K.B. and Neumann. M.M., 2000. An Introduction to Local Spectral Theory, Mathematical Society Monographs New Series, 20, Clarendon Press, Oxford, London, pp (14-222) .

2. Campbell, S.L. 1975. Linear operators for which $T^{*} T$ and $T+T^{*}$ commute, Pacific. J. Math., 61: $53-57$.

3. Conway, 1985. A course in functional analysis, SpringerVerlage, Berlin Heidelberg, New York, pp (39-197).

4. Campbell, S.L. and Gellar. R.1976. Spectral properties of linear operators for which $T^{*} T$ and $T+T^{*}$ commute, Proc. Amer. Math. Soc., 60: 197-202.

5. Campbell, S.L. and R. Gellar. 1977. linear operators for which $T^{*} T$ and $T+T^{*}$ commute II. Trans. Amer. Math. Soc., 226: 305-319.

6. Al-Saltan, R. E. 2000. Some generalizations of normal operators, M. Sc. Thesis, College of Science, University of Baghdad, pp (46-48)

7. Feldman, N.S., T.L. Miller, and V.G. Miller. 2002. Hypercyclic and 
Supercyclic Cohyponormal Operators, Acta Sci. Math. (Szeged), 68: 303-328.

8. Feldman, N.S. 2003. Countably Hypercyclic Operators, J. Operator Theory, 50: 107-117.

9. Feldman, N.S.2002. ,The dynamics of cohyponormal operators, Contemp. Math. 321, Amer. Math. Soc.: 71-82.
10. Y. Kato. 1994. Some examples of $\theta$-operators, Kyushu Journal of Mathematics, 48: 101-109

11. Uchiyama, A. and Tanahashi, $K$. 2007. Some spectral properties which imply Bishop's property $(\beta)$, RIMS Kokyuroku, 1535: 143148.

12. Shaakir. L.K. 1999. Cyclic operators on Hilbert spaces, M. Sc. Thesis, College of Science, University of Baghdad, pp (57)

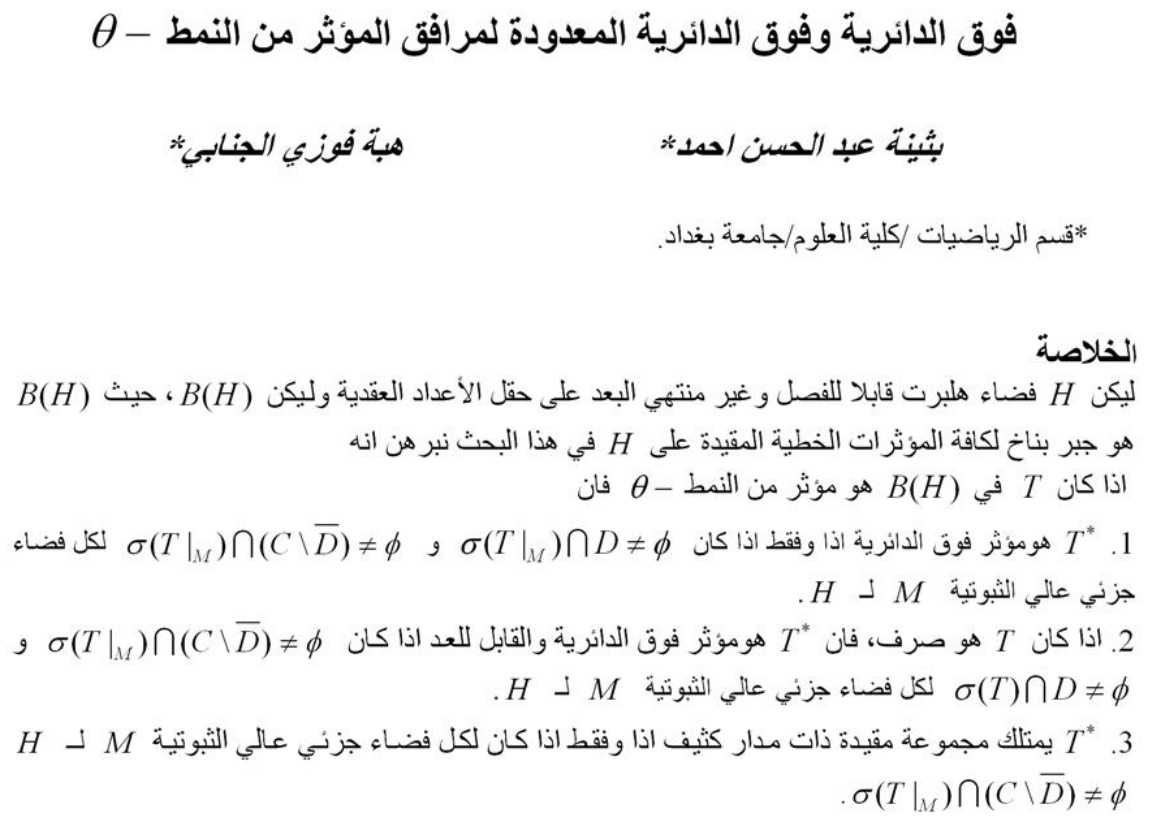

